\title{
Habitual sugar intake and cognitive function among middle-aged and older Puerto Ricans without diabetes
}

\author{
Xingwang $\mathrm{Ye}^{1,2}$, Xiang Gao ${ }^{3,4}$, Tammy $\mathrm{Scott}^{5}$ and Katherine L. Tucker ${ }^{1,2 *}$ \\ ${ }^{1}$ Jean Mayer US Department of Agriculture Human Nutrition Research Center on Aging at Tufts University, Boston, MA, USA \\ ${ }^{2}$ Department of Health Sciences, Northeastern University, Boston, MA, USA \\ ${ }^{3}$ Department of Nutrition, Harvard University School of Public Health, Boston, MA, USA \\ ${ }^{4}$ Channing Laboratory, Department of Medicine, Brigham and Women's Hospital, and Harvard Medical School, Boston, MA, USA \\ ${ }^{5}$ Department of Psychiatry, Tufts Medical Center, Boston, MA, USA
}

(Received 15 September 2010 - Revised 28 February 2011 - Accepted 9 March 2011 - First published online 1 June 2011)

\section{Abstract}

Intake of added sugars, mainly fructose and sucrose, has been associated with risk factors for cognitive impairment, such as obesity, the metabolic syndrome and type 2 diabetes. The objective of this analysis was to examine whether habitual intakes of total sugars, added sugars, sugar-sweetened beverages or sweetened solid foods are associated with cognitive function. The present study included 737 participants without diabetes, aged 45-75 years, from the Boston Puerto Rican Health Study, 2004-9. Cognitive function was measured with a battery of seven tests: Mini-Mental State Examination (MMSE), word list learning, digit span, clock drawing, figure copying, and Stroop and verbal fluency tests. Usual dietary intake was assessed with a validated FFQ. Greater intakes of total sugars, added sugars and sugarsweetened beverages, but not of sugar-sweetened solid foods, were significantly associated with lower MMSE score, after adjusting for covariates. Adjusted OR for cognitive impairment (MMSE score <24) were 2.23 (95\% CI 1.24, 3.99) for total sugars and $2 \cdot 28$ (95\% CI $1 \cdot 26,4 \cdot 14)$ for added sugars, comparing the highest with lowest intake quintiles. Greater intake of total sugars was also significantly associated with lower word list learning score. In conclusion, higher sugar intake appears to be associated with lower cognitive function, but longitudinal studies are needed to clarify the direction of causality.

\section{Key words: Added sugar: Sugar-sweetened beverages: Cognition function}

Energy from sugar-sweetened beverages (SSB) has increased in the USA during the last three decades ${ }^{(1)}$. Paralleling this trend has been the well-known increased prevalence of obesity $^{(2)}$. In particular, beverages sweetened with high-fructose corn syrup (HFCS) have been implicated in relation to the development of obesity ${ }^{(3)}$, and longitudinal data support the notion that SSB consumption is associated with increased $\mathrm{BMI}^{(4)}$. Emerging data have also linked SSB intake to central obesity $^{(5)}$, high blood pressure ${ }^{(5-7)}$, dyslipidaemia ${ }^{(5)}$ and a constellation of these metabolic risk factors, i.e. the metabolic syndrome ${ }^{(5,8)}$. In addition, SSB intake has been associated with the incidence of type 2 diabetes ${ }^{(8-10)}$. Recently, the metabolic syndrome has been linked with cognitive impairment cross-sectionally $^{(11)}$ and prospectively ${ }^{(12-14)}$. Type 2 diabetes is also considered to be a risk factor for impaired cognitive function $^{(15)}$. However, it remains unclear whether habitual consumption of SSB is associated with cognitive dysfunction.

HFCS has gained much attention, because food industry sweeteners have shifted from sucrose to $\mathrm{HFCS}^{(16)}$. Limited data from animal studies suggest that HFCS may have a role in cognitive impairment. Stranahan et $a l .{ }^{(17)}$ found that rats fed a high-fat, high-glucose diet supplemented with HFCS showed impaired hippocampal synaptic and cognitive function, possibly via development of insulin resistance ${ }^{(17)}$ Hamsters fed a high-fructose diet have been shown to exhibit insulin resistance in the hippocampus, but cognitive function in these animals has not been examined ${ }^{(18)}$. Nevertheless, due to the similar composition of widely used HFCS to sucrose $^{(19)}$, it remains unclear whether cognitive function is associated with HFCS, with sucrose, or with both.

Although studies have been conducted to investigate immediate or short-term effects of pure glucose and other types of carbohydrates on cognitive performance ${ }^{(20)}$, to our knowledge no epidemiological studies have examined associations of habitual consumption of fructose and other sugars with cognitive function among middle-aged and older adults $^{(21)}$. In addition, it remains unclear whether SSB differ in their associations with cognitive function, relative to

Abbreviations: HFCS, high-fructose corn syrup; MMSE, Mini-Mental State Examination; SSB, sugar-sweetened beverage.

*Corresponding author: Dr Katherine L. Tucker, email kl.tucker@neu.edu 
sugar-sweetened solid foods or 100\% fruit juice. In the present study we, therefore, examined habitual intakes of total sugars, added sugars, SSB, $100 \%$ fruit juice and sugar-sweetened solid foods, as well as sucrose and added fructose, and their associations with cognitive function among a subsample of Puerto Rican adults without diabetes, living in the greater Boston area.

\section{Materials and methods}

\section{Participants}

The Boston Puerto Rican Health Study is an ongoing study funded by the National Institutes of Health as one of their Centers for Population Health and Health Disparities ${ }^{(22)}$. Detailed information regarding the design of the study has been described previously ${ }^{(22)}$. Briefly, data collection began in 2004 and was completed in 2009. Census tracts containing at least twenty-five Puerto Rican adults, aged 45-75 years, in the year 2000 census were identified in the greater Boston area. All blocks with ten or more Hispanic adults of the same age range were enumerated. One Puerto Rican adult, aged 45-75 years, per household was randomly recruited from qualified households in selected blocks. Most (77.4\%) participants were recruited this way, with an additional $9.8 \%$ identified through partnerships with community organisations and random approach at major community events, $7 \cdot 2 \%$ from referrals from community members and 5.6\% from calls responding to posted flyers. A total of 2170 individuals were identified. Of these, seventy-seven participants were excluded because they were unable to answer questions due to a serious health condition or advanced dementia, they planned to move from the area within 2 years, or they did not have a permanent address. All others ( $n$ 2093) were invited to participate and $1811(86.5 \%)$ agreed to be interviewed. After excluding nine participants with low Mini-Mental State Examination (MMSE) score $(\leq 10), 1802$ participants were eligible to continue the survey. A total of 1500 (83.2\%) individuals finally completed the baseline interview whereas others ( $n$ 302) did not complete the interview due to difficulty in scheduling, or unsuccessful follow-up. Cleaned data for cognitive tests, dietary intake and plasma biomarkers were available for 1300 participants at the time of analysis. The present study was conducted according to the guidelines laid down in the Declaration of Helsinki and all procedures involving human subjects were approved by the Institutional Review Board at Tufts Medical Center. Written informed consent was obtained from all participants. In the present study, we excluded participants ( $n$ 520) who took medications for diabetes or those who had fasting glucose $\geq 7.0 \mathrm{mmol} / \mathrm{l}$, because diabetes has been linked to cognitive impairment ${ }^{(15)}$, and because those with diabetes may change their dietary pattern, especially intake of added sugars. We also excluded participants reporting implausible energy consumption (<2512 kJ (600 kcal) per d and >20 $093 \mathrm{~kJ}$ (4800 kcal) per d) ( $n$ 43), resulting in 737 participants in the present analyses.

During the home interview, data about social-demographic status, lifestyle, dietary intake, cognitive function, self-reported diagnosed health conditions and medication use were collected by bilingual trained research assistants in Spanish or English depending on the primary language spoken by the participant at home.

\section{Assessment of usual food and nutrient consumption}

Usual food and nutrient intakes were estimated for the last 12 months from a semi-quantitative FFQ with 246 food items ${ }^{(23)}$. This FFQ has been validated against plasma carotenoids ${ }^{(24)}$ and vitamin $\mathrm{B}_{12}{ }^{(25)}$ in Hispanics including Puerto Ricans aged 60 years and over. Nutrient intakes were calculated using the Nutrition Data System for Research software version 2007 developed from the Food and Nutrient Database 2007 (Nutrition Coordinating Center, University of Minnesota, Minneapolis, MN, USA).

Total sugars were defined as the sum of three free monosaccharides (glucose, fructose and galactose) and three free disaccharides (sucrose, lactose and maltose). Data for these mono- and disaccharides in the nutrient database include those from naturally occurring sources, such as fruit and vegetables, and from added sources, such as sucrose and HFCS. Added sugars included sugars and syrups added to foods during food preparation or commercial food processing ${ }^{(26)}$, and did not include mono- and disaccharides occurring naturally in foods, such as lactose in milk or fructose in fruit. Ingredients designated as added sugars were white sugar (sucrose), brown sugar, powdered sugar, honey, molasses, pancake syrup, corn syrup, malt syrup, fructose, glucose (dextrose), galactose and lactose ${ }^{(26)}$.

In the FFQ, nineteen items were designed to collect information about intakes of specific types of beverages, including $100 \%$ fruit juice, fruit nectars, fruit drinks, soft drinks and teas. In the present study, SSB included regular fruit drinks with added sugars (fruit drinks, fruit juices and fruit nectars) and soft drinks (carbonated beverages with added sugars). Sugar-sweetened solid foods included sweets (candy, cookies, cakes, pies, doughnuts, sweet rolls, energy or granola bars) and dairy desserts (ice cream, sherbet, frozen yogurt and pudding). Included in $100 \%$ fruit juice were fruit juices (orange, apple and other) with no added sugars. We defined naturally occurring fructose as fructose from fruit and fruit products without added sugars (such as 100\% fruit juice, fresh, frozen, canned and dried fruits) and vegetables and vegetable products without added sugars (for example, onions, peppers, tomatoes and canned plain tomato sauces). Fructose from other sources was defined as added fructose.

\section{Assessment of cognitive function}

As described previously, seven cognitive tests were administered to each participant in their home on the same day that dietary intake was assessed with the FFQ by a qualified research assistant ${ }^{(27)}$. Briefly, the MMSE was administered to assess general cognitive function ${ }^{(28)}$ (scores ranged from 12 to 30 in the present study). Also administered were: a sixteen-word list learning task to assess verbal memory, with subscores including learning and immediate recall (the sum 
of words recalled over five learning trials), recognition (the number of words discriminated correctly from a longer word list after a $25-35$ min delay) and percentage retention (calculated by dividing the number of words recalled after the delay by the number of correct responses on the fifth learning trial) ${ }^{(29)}$; digit span forward and backward, to assess attention and working memory ${ }^{(29)}$; the Stroop test, to measure cognitive flexibility, response inhibition and processing speed ${ }^{(29)}$; verbal fluency, to assess the speed at which one can provide exemplars to a category (initial letter of a word) ${ }^{(29)}$; and clock drawing $^{(30)}$ and figure copying ${ }^{(31)}$, both to assess visual-spatial organisation. Scores for figure copying were weighted for the complexity of the figure copied; one point for easy figures and four points for the most difficult. Higher scores on each of these seven tests signify better cognition.

Factor analysis was used to conduct data reduction based on the scores of the seven cognitive tests, as described previously $^{(27)}$. We identified three factors with Kaiser's criterion (Eigenvalue $>1)^{(32)}$. A varimax rotation was performed to improve the interpretation of these factors. These factors were then labelled as memory, executive function and attention, respectively (see Supplemental Table 1; available at http://www.journals.cambridge.org/bjn). In the present study, the MMSE scores were used as the primary outcome because the MMSE test reflects general cognitive function. We also examined whether intakes of total and added sugars were associated with the three derived cognitive function factors and with scores from individual tests.

\section{Assessment of covariates}

Educational attainment was categorised into five groups by their highest degree (below 5th grade, 5th-8th grade, 9th12 th grade, college, or graduate school). Smoking status was categorised as never smoking $(<100$ cigarettes in entire life), former smoking or current smoking. Alcohol use was classified as not current, current moderate $(\leq$ one drink per $\mathrm{d}$ for women and $\leq$ two drinks per $\mathrm{d}$ for men) or current heavy ( $>$ one drink per $\mathrm{d}$ for women and $>$ two drinks per $\mathrm{d}$ for men). Physical activity was estimated as a score, based on a modified Paffenbarger questionnaire of the Harvard Alumni Activity Survey ${ }^{(33)}$. The score was constructed by weighting time spent in various physical activities by factors that reflect $\mathrm{O}_{2}$ consumption of related physical activities. Poverty was defined as 'yes' if a participant's total annual house income was below the threshold released each year by the US Department of Health and Human Services. Acculturation score was calculated based on answers to seven questions regarding the extent of use of English and/or Spanish at work, in watching television, listening to the radio, reading newspapers/books, speaking with neighbours, talking to friends and talking to family members: a summed score ranged from 0 (fully unacculturated, only using Spanish) to 100 (fully acculturated, only using English) ${ }^{(34)}$.

Anthropometric measures were taken in the home with standard methods ${ }^{(23)}$. BMI was calculated as weight in kilograms divided by squared height in metres. Blood pressure was measured at three time points during the home interview, and the latter two measures were averaged. Hypertension was defined as systolic blood pressure $\geq 140 \mathrm{mmHg}$ or diastolic blood pressure $\geq 90 \mathrm{mmHg}$ or use of antihypertensive medications.

\section{Statistical analyses}

Intakes of total sugar, added sugar, specific sugars, types of beverages and food were adjusted for total energy intake using the regression residual method ${ }^{(35)}$. For example, adjusted total sugar intake was the sum of the regression residuals and a constant equal to the expected total sugar intake at the mean total energy intake. The same procedure was used to calculate adjusted values for other types of sugars, beverages and food before categorisation into quintiles for statistical analyses. General linear models were used to calculate adjusted means of cognitive scores by quintile of energy-adjusted sugar consumption, after controlling for age, sex, educational attainment (below 5th grade, 5th-8th grade, 9th-12th grade, college, or graduate school), poverty (yes or no), smoking (never, former, or current), alcohol use (not current, current moderate, current heavy), physical activity score, BMI $\left(\mathrm{kg} / \mathrm{m}^{2} ;<25,25-29 \cdot 9\right.$, or $\left.\geq 30\right)$ and presence of hypertension. Acculturation score was also adjusted, because language use (Spanish $v$. English) might influence the results of cognitive tests ${ }^{(36)}$ and also be related to dietary intake ${ }^{(37)}$. Means were compared with Tukey's adjustment for multiple comparisons. In secondary analyses, we further adjusted for intake of dietary fibre, total cholesterol, fat (saturated, monounsaturated, polyunsaturated and trans), vitamin supplement use (yes or no) and plasma concentrations of vitamin $\mathrm{C}$, total carotenoids and total homocysteine. Because there are no well-established age- and educationbased cut-off points for cognitive impairment specifically for this population, we used logistic regression models to estimate OR for cognitive impairment defined by the traditional cut-off point of 24 of the MMSE score (MMSE score <24) in the upper four quintiles relative to the first quintile of total and added sugar intakes, respectively. Because a large proportion of individuals (45.3\%) had an education of less than 9 years, we repeated our analyses using a modified definition of cognitive impairment (MMSE score $<21)^{(38)}$. Continuous values of sugar consumption were used for trend tests. All statistical analyses were conducted with SAS (version 9.1.3; SAS Institute Inc., Cary, NC, USA). A value of $P<0.05$ (two-sided) was considered statistically significant.

\section{Results}

Among participants without diabetes (mean age 56.3 (SD 7.6) years), the averages of total and added sugar intakes were 115 (SD 62) and 64.0 (SD 45.4) g/d, respectively. Total sugars contributed to 21.4 (SD 8.0 ) \% of energy intake, and added sugars contributed to 11.8 (SD 8.0 ) \%. Sucrose was the most consumed sugar (46.7 (SD 27.9) g/d), followed by fructose $(25 \cdot 2(\mathrm{SD} 19 \cdot 3) \mathrm{g} / \mathrm{d})$ and glucose $(25 \cdot 0$ (SD 17.9) g/d). Together, sucrose, glucose and fructose contributed $84.4 \%$ of total sugar consumption. Fruit drinks, soft drinks, dairy desserts and 
sweets provided $22 \cdot 1,12 \cdot 9,11 \cdot 3$ and $10 \cdot 3 \%$ of added sugars, respectively. The mean MMSE score was 23.7 (SD 3.4). Approximately $47 \cdot 0 \%$ had a MMSE score $<24$ and $16.7 \%$ had a MMSE score $<21$ in this population.

Participants in the highest quintile of energy-adjusted total sugar intake were more likely to be older, women, and to have lower prevalence of heavy drinkers, lower BMI, lower prevalence of hypertension, and higher plasma vitamin $\mathrm{C}$ concentration, relative to participants with the lowest energy-adjusted total sugar consumption (Table 1). As expected, participants in the highest quintile of energyadjusted total sugar consumption had significantly lower intake of fat, but higher intakes of added sugars, sucrose, glucose, natural and added fructose, galactose, maltose, lactose, maltose, SSB, sugar-sweetened solid foods and $100 \%$ fruit juice (all $P<0.05$; Table 2 ) when compared with those in the lowest quintile of energy-adjusted total sugar consumption.
Intake of total sugars was strongly associated with intakes of added sugars, sucrose, glucose, added fructose and SSB ( $r 0.63$ to 0.83 ; all $P<0.001)$, moderately associated with natural fructose and $100 \%$ fruit juice $(r 0.43$ and 0.46 , respectively; $P<0 \cdot 001)$ and weakly associated with sugar-sweetened solid foods, lactose, maltose and galactose ( $r \quad 0 \cdot 15$ to $0 \cdot 30$; all $P<0.001$ ) (see Supplemental Table 2; available at http:// www.journals.cambridge.org/bjn).

Total and added sugar intakes were each inversely associated with MMSE score, after adjusting for several covariates (Table 3). The MMSE score was 0.4 points lower for each $60 \mathrm{~g}$ of total sugar intake $(\beta-0.41$; SE 0.15 ; $P=0.007)$, after adjusting for age, sex, educational attainment, poverty, acculturation score, smoking, alcohol use, physical activity score, BMI and the presence of hypertension. This difference in MMSE score was approximately comparable with an associated difference in MMSE score per 10-year increase in age ( $\beta-0.39$; SE $0 \cdot 17 ; P=0.020$ ) in the same model. The MMSE

Table 1. Characteristics of participants without diabetes from the Boston Puerto Rican health study by quintile of energy-adjusted total sugar intake, 2004-9†

(Mean values with their standard errors or percentages)

\begin{tabular}{|c|c|c|c|c|c|c|c|c|c|c|c|}
\hline \multirow[b]{3}{*}{ Variable } & \multicolumn{10}{|c|}{ Quintile of energy-adjusted total sugar intake } & \multirow{3}{*}{$\begin{array}{l}P \text { for } \\
\text { trend } \neq\end{array}$} \\
\hline & \multicolumn{2}{|c|}{$\begin{array}{l}\text { 1st quintile } \\
(<82.0 \mathrm{~g} / \mathrm{d})\end{array}$} & \multicolumn{2}{|c|}{$\begin{array}{l}\text { 2nd quintile } \\
(82.0- \\
100 \cdot 8 \mathrm{~g} / \mathrm{d})\end{array}$} & \multicolumn{2}{|c|}{$\begin{array}{c}\text { 3rd quintile } \\
(100.9- \\
118.3 \mathrm{~g} / \mathrm{d})\end{array}$} & \multicolumn{2}{|c|}{$\begin{array}{c}\text { 4th quintile } \\
(118.4- \\
145.5 \mathrm{~g} / \mathrm{d})\end{array}$} & \multicolumn{2}{|c|}{$\begin{array}{l}\text { 5th quintile } \\
(>145.5 \mathrm{~g} / \mathrm{d})\end{array}$} & \\
\hline & Mean & SE & Mean & SE & Mean & SE & Mean & SE & Mean & SE & \\
\hline Subjects $(n)$ & \multicolumn{2}{|c|}{147} & \multicolumn{2}{|c|}{148} & \multicolumn{2}{|c|}{147} & \multicolumn{2}{|c|}{148} & \multicolumn{2}{|c|}{147} & \\
\hline Total sugars $(\mathrm{g} / \mathrm{d})$ & $59 \cdot 2$ & $1 \cdot 8$ & 91.7 & 0.4 & $109 \cdot 9$ & 0.4 & $130 \cdot 5$ & 0.6 & $182 \cdot 6$ & 3.5 & \\
\hline Age (years) & $54 \cdot 1$ & 0.6 & $56 \cdot 7^{\star}$ & 0.6 & $57 \cdot 4^{\star \star}$ & 0.6 & $57 \cdot 1^{\star \star}$ & 0.7 & $56 \cdot 9^{\star \star}$ & 0.6 & 0.006 \\
\hline Female $(\%)$ & \multicolumn{2}{|c|}{55.8} & \multicolumn{2}{|c|}{$73 \cdot 0^{\star *}$} & \multicolumn{2}{|c|}{$72 \cdot 8^{\star \star}$} & \multicolumn{2}{|c|}{$82 \cdot 4^{\star \star}$} & \multicolumn{2}{|c|}{$76 \cdot 9^{\star \star \star}$} & $<0.001$ \\
\hline Poverty (yes, \%)§ & \multicolumn{2}{|c|}{$61 \cdot 2$} & \multicolumn{2}{|c|}{$56 \cdot 1$} & \multicolumn{2}{|c|}{$60 \cdot 1$} & \multicolumn{2}{|c|}{$53 \cdot 2^{*}$} & \multicolumn{2}{|c|}{$56 \cdot 4$} & 0.36 \\
\hline Acculturation scorell & $25 \cdot 0$ & 1.8 & 24.9 & 1.8 & $28 \cdot 5$ & 1.8 & $29 \cdot 1$ & 1.9 & $29 \cdot 1$ & 1.9 & 0.10 \\
\hline Education (\%) & & & & & & & & & & & 0.82 \\
\hline Below 5th grade & \multicolumn{2}{|c|}{$14 \cdot 3$} & \multicolumn{2}{|c|}{$25 \cdot 2$} & \multicolumn{2}{|c|}{23.8} & \multicolumn{2}{|c|}{$19 \cdot 7$} & \multicolumn{2}{|c|}{20.4} & \\
\hline 5th-8th grade & & & & & 27 & & & & & & \\
\hline 9th-12th grade & & & & & 33 & & & & & & \\
\hline College & & & & & 11 & & & & & & \\
\hline Graduate school & & & & & 3 & & & & & & \\
\hline Smoking (\%) & & & & & & & & & & & 0.92 \\
\hline Never & & & & & 40 & & & & & & \\
\hline Former & & & & & 33 & & & & & & \\
\hline Current & & & & & 26 & & & & & & \\
\hline Alcohol use (\%) & & & & & & & & & & & 0.004 \\
\hline Not currently & & & & & 56 & & & & & & \\
\hline Current moderate & & & & & 37 & & & & & & \\
\hline Current heavy & & & & & $5 \cdot 5$ & & & & & & \\
\hline Physical activity score & $32 \cdot 1$ & 0.4 & 31.9 & 0.4 & 31.8 & 0.4 & $32 \cdot 2$ & 0.4 & $32 \cdot 6$ & 0.4 & 0.71 \\
\hline $\mathrm{BMI}\left(\mathrm{kg} / \mathrm{m}^{2}\right)$ & $31 \cdot 0$ & 0.5 & $30 \cdot 2$ & 0.5 & $30 \cdot 1$ & 0.5 & $30 \cdot 0$ & 0.5 & $28 \cdot 7^{\star *}$ & 0.5 & 0.064 \\
\hline Hypertension (yes, \%) & & & & & 60 & & & & & & 0.014 \\
\hline Plasma homocysteine $(\mu \mathrm{mol} / \mathrm{l})$ & 9.9 & 0.4 & $9 \cdot 4$ & 0.4 & 8.8 & 0.4 & $9 \cdot 5$ & 0.4 & $9 \cdot 3$ & 0.4 & 0.66 \\
\hline Plasma vitamin $\mathrm{C}(\mu \mathrm{mol} / \mathrm{l})$ & $42 \cdot 7$ & 1.8 & $49 \cdot 3$ & $1 \cdot 8$ & $52 \cdot 8^{\star \star \star}$ & $1 \cdot 8$ & $48 \cdot 6$ & 1.8 & $50 \cdot 9^{\star}$ & $1 \cdot 8$ & 0.041 \\
\hline Plasma total carotenoids $(\mu \mathrm{mol} / \mathrm{l})$ & 1.87 & 0.06 & 1.85 & 0.06 & 1.77 & 0.06 & 1.80 & 0.06 & 1.71 & 0.06 & 0.009 \\
\hline Vitamin supplement use (\%) & & & & & 64 & & & & & & 0.88 \\
\hline
\end{tabular}

Value was significantly different from that of the first quintile: ${ }^{\star} P<0.05,{ }^{\star \star} P<0.01,{ }^{\star \star \star} P<0.001$.

$\dagger$ Values for continuous variables were adjusted for age (years) and sex. Means were compared using the general linear model procedure (PROC GLM) in SAS, with Tukey's adjustment for multiple comparisons. Percentages of categorical variables across quintiles of total sugar intake were compared using logistic regression (PROC LOGISTIC) in SAS after adjustment for age (years) and sex.

$\ddagger$ Continuous value of total sugar intake was used for the trend test.

$\S$ Poverty was defined as 'yes' if a participant's total annual house income was below the poverty threshold released each year by the US Department of Health and Human Services. || Acculturation score was calculated based on answers of questions regarding the extent of use of English and/or Spanish at work and in ordinary life: a summed score ranged from 0 (fully unacculturated: only using Spanish) to 100 (fully acculturated: only using English).

I Physical activity score was constructed by weighting time spent in various physical activities by factors that reflect $\mathrm{O}_{2}$ consumptions of related physical activities. 
Table 2. Intakes of selected nutrients, sugars and food by quintile of energy-adjusted total sugar intake in participants without diabetes from the Boston Puerto Rican health study, 2004-9† (Mean values with their standard errors)

\begin{tabular}{|c|c|c|c|c|c|c|c|c|c|c|c|}
\hline \multirow[b]{3}{*}{ Variable } & \multicolumn{10}{|c|}{ Quintile of energy-adjusted total sugar intake } & \multirow{3}{*}{$\begin{array}{l}P \text { for } \\
\text { trend }\end{array}$} \\
\hline & \multicolumn{2}{|c|}{$\begin{array}{l}\text { 1st quintile } \\
(<82.0 \mathrm{~g} / \mathrm{d})\end{array}$} & \multicolumn{2}{|c|}{$\begin{array}{l}\text { 2nd quintile } \\
(82.0-100.8 \mathrm{~g} / \mathrm{d})\end{array}$} & \multicolumn{2}{|c|}{$\begin{array}{c}\text { 3rd quintile } \\
(100.9-118.3 \mathrm{~g} / \mathrm{d})\end{array}$} & \multicolumn{2}{|c|}{$\begin{array}{c}\text { 4th quintile } \\
(118.4-145.5 \mathrm{~g} / \mathrm{d})\end{array}$} & \multicolumn{2}{|c|}{$\begin{array}{c}\text { 5th quintile } \\
(>145.5 \mathrm{~g} / \mathrm{d})\end{array}$} & \\
\hline & Mean & $\mathrm{SE}$ & Mean & SE & Mean & SE & Mean & SE & Mean & SE & \\
\hline Total energy intake $(\mathrm{kJ} / \mathrm{d})$ & 11239 & 285 & $8289^{\star \star *}$ & 289 & $8108^{\star \star *}$ & 290 & $9078^{\star \star \star}$ & 296 & 10609 & 293 & 0.27 \\
\hline Dietary fibre intake $(\mathrm{g} / \mathrm{d})$ & $21 \cdot 2$ & 0.5 & $21 \cdot 2$ & 0.5 & 21.5 & 0.5 & 20.5 & 0.5 & 20.4 & 0.5 & 0.046 \\
\hline Saturated fat intake $(\mathrm{g} / \mathrm{d})$ & $24 \cdot 3$ & 0.5 & 24.5 & 0.5 & 23.5 & 0.5 & 23.6 & 0.5 & $22 \cdot 2^{*}$ & 0.5 & $<0.001$ \\
\hline Polyunsaturated fat intake (g/d) & 24.4 & 0.4 & $22 \cdot 4^{\star \star}$ & 0.4 & $21.5^{\star \star \star}$ & 0.4 & $19 \cdot 6^{\star \star \star}$ & 0.4 & $16 \cdot 7^{\star \star \star}$ & 0.4 & $<0.001$ \\
\hline Trans-fat intake $(\mathrm{g} / \mathrm{d})$ & 2.75 & 0.08 & 2.75 & 0.08 & 2.76 & 0.08 & 2.70 & 0.08 & 2.45 & 0.08 & 0.005 \\
\hline Added sugars $(\mathrm{g} / \mathrm{d})$ & $29 \cdot 3$ & $2 \cdot 2$ & $50 \cdot 5^{\star \star \star}$ & $2 \cdot 3$ & $58 \cdot 7^{\star \star \star}$ & $2 \cdot 3$ & $73 \cdot 6^{\star \star \star}$ & $2 \cdot 3$ & $109 \cdot 8^{\star \star \star}$ & $2 \cdot 3$ & $<0.001$ \\
\hline Sucrose $(g / d)$ & 23.9 & 1.4 & $39 \cdot 1^{\star * *}$ & 1.4 & $45 \cdot 6^{\star \star \star}$ & 1.4 & $55 \cdot 4^{\star * *}$ & 1.4 & $71.9^{\star \star \star}$ & 1.4 & $<0.001$ \\
\hline Glucose $(\mathrm{g} / \mathrm{d})$ & $12 \cdot 7$ & 0.9 & $19 \cdot 1^{\star \star \star}$ & 0.9 & $22 \cdot 9^{\star \star \star}$ & 1.0 & $27 \cdot 7^{\star \star \star}$ & $1 \cdot 0$ & $42 \cdot 4^{\star \star \star}$ & 1.0 & $<0.001$ \\
\hline Fructose $(\mathrm{g} / \mathrm{d})$ & 11.4 & 1.0 & $18 \cdot 3^{\star \star \star}$ & 1.1 & $22 \cdot 9^{\star \star \star}$ & $1 \cdot 1$ & $28 \cdot 4^{\star \star *}$ & $1 \cdot 1$ & $44 \cdot 7^{\star \star \star}$ & $1 \cdot 1$ & $<0.001$ \\
\hline Natural fructose $(\mathrm{g} / \mathrm{d})$ & $6 \cdot 3$ & 0.7 & $9 \cdot 1^{*}$ & 0.7 & $11 \cdot 6^{\star * *}$ & 0.7 & $13 \cdot 9^{* \star *}$ & 0.7 & $18 \cdot 3^{\star \star *}$ & 0.7 & $<0.001$ \\
\hline Added fructose $(\mathrm{g} / \mathrm{d})$ & $5 \cdot 1$ & 1.0 & $9 \cdot 2^{*}$ & 1.0 & $11 \cdot 3^{\star \star \star}$ & 1.0 & $14 \cdot 5^{\star \star \star}$ & $1 \cdot 0$ & $26 \cdot 4^{\star \star \star}$ & 1.0 & $<0.001$ \\
\hline Galactose $(\mathrm{g} / \mathrm{d})$ & 0.30 & 0.07 & 0.51 & 0.07 & 0.53 & 0.07 & 0.49 & 0.07 & $0.70^{\star \star \star}$ & 0.07 & $<0.001$ \\
\hline Lactose $(\mathrm{g} / \mathrm{d})$ & $9 \cdot 4$ & 0.9 & $12 \cdot 4$ & 0.9 & $15 \cdot 7^{\star \star \star}$ & 0.9 & $16 \cdot 1^{\star \star \star}$ & 0.9 & $20 \cdot 2^{\star \star \star}$ & 0.9 & $<0.001$ \\
\hline Maltose $(\mathrm{g} / \mathrm{d})$ & 1.6 & 0.1 & $2 \cdot 2^{\star \star \star}$ & 0.1 & $2 \cdot 1^{\star \star}$ & 0.1 & $2 \cdot 3^{\star \star \star}$ & 0.1 & $2 \cdot 6^{\star \star \star}$ & 0.1 & $<0.001$ \\
\hline Sugar-sweetened beverages $(\mathrm{g} / \mathrm{d})$ & 73.9 & $20 \cdot 3$ & $130 \cdot 8$ & $20 \cdot 6$ & $173 \cdot 1^{\star \star}$ & $20 \cdot 7$ & $242 \cdot 7^{\star \star *}$ & $21 \cdot 1$ & $478 \cdot 5^{\star \star \star}$ & $20 \cdot 9$ & $<0.001$ \\
\hline Sugar-sweetened solid foods ( $\mathrm{g} / \mathrm{d}$ ) & $28 \cdot 1$ & 4.7 & $46 \cdot 0$ & 4.7 & $58 \cdot 6^{\star \star \star}$ & 4.7 & $68 \cdot 2^{\star \star \star}$ & 4.8 & $75 \cdot 4^{\star \star \star}$ & 4.8 & $<0.001$ \\
\hline $100 \%$ fruit juice $(\mathrm{g} / \mathrm{d})$ & $59 \cdot 1$ & $15 \cdot 7$ & $125 \cdot 8^{*}$ & $15 \cdot 9$ & $186 \cdot 4^{\star \star \star}$ & $16 \cdot 0$ & $234 \cdot 4^{\star \star \star}$ & $16 \cdot 3$ & $339 \cdot 9^{\star \star \star}$ & $16 \cdot 8$ & $<0.001$ \\
\hline
\end{tabular}

Mean value was significantly different from that of the first quintile: ${ }^{*} P<0.05,{ }^{\star \star} P<0.01,{ }^{\star \star \star} P<0.001$

† Values were adjusted for age (years) and sex. Means were compared using the general linear model procedure (PROC GLM) in SAS, with Tukey's adjustment for multiple comparisons.

$\ddagger$ Continuous value of total sugar intake was used for the trend test. 
Table 3. Mini-Mental State Examination scores by quintile of energy-adjusted sugar and food intake in participants without diabetes from the Boston Puerto Rican Health Study, 2004-9†

(Mean values with their standard errors)

\begin{tabular}{|c|c|c|c|c|c|c|c|c|c|c|c|}
\hline \multirow[b]{2}{*}{ Variable } & \multicolumn{2}{|c|}{ 1st quintile } & \multicolumn{2}{|c|}{ 2nd quintile } & \multicolumn{2}{|c|}{ 3rd quintile } & \multicolumn{2}{|c|}{ 4th quintile } & \multicolumn{2}{|c|}{ 5th quintile } & \multirow{2}{*}{$\begin{array}{l}P \text { for } \\
\text { trendf }\end{array}$} \\
\hline & Mean & SE & Mean & SE & Mean & SE & Mean & SE & Mean & SE & \\
\hline Total sugars & $25 \cdot 0$ & 0.3 & $25 \cdot 0$ & 0.3 & $24 \cdot 6$ & 0.3 & $24 \cdot 6$ & 0.3 & $24 \cdot 1$ & 0.3 & 0.007 \\
\hline Added sugars & $25 \cdot 4$ & 0.3 & $24 \cdot 6$ & 0.3 & $24 \cdot 2^{*}$ & 0.3 & 24.5 & 0.3 & $24 \cdot 2^{*}$ & 0.3 & 0.005 \\
\hline Sucrose & $25 \cdot 1$ & 0.3 & $24 \cdot 8$ & $0 \cdot 3$ & 24.4 & 0.3 & 24.5 & $0 \cdot 3$ & $24 \cdot 3$ & $0 \cdot 3$ & 0.014 \\
\hline Glucose & $24 \cdot 8$ & 0.3 & $25 \cdot 1$ & 0.3 & $25 \cdot 1$ & 0.3 & 24.4 & 0.3 & $24 \cdot 2$ & 0.3 & 0.032 \\
\hline Fructose & 24.9 & 0.3 & $25 \cdot 3$ & 0.3 & 24.9 & 0.3 & $24 \cdot 3$ & 0.3 & $24 \cdot 3$ & 0.3 & 0.037 \\
\hline Natural fructose & 24.4 & 0.3 & $25 \cdot 1$ & 0.3 & $25 \cdot 1$ & 0.3 & $25 \cdot 0$ & 0.3 & 24.4 & 0.3 & 0.61 \\
\hline Added fructose§ & $25 \cdot 1$ & 0.3 & $25 \cdot 1$ & 0.3 & $25 \cdot 1$ & 0.3 & $24 \cdot 3$ & 0.3 & $24 \cdot 3$ & 0.3 & 0.028 \\
\hline Galactose & $24 \cdot 2$ & 0.3 & $25 \cdot 2^{*}$ & 0.3 & 24.9 & 0.3 & 24.9 & 0.3 & 24.5 & 0.3 & 0.99 \\
\hline Lactose & $25 \cdot 0$ & 0.3 & 24.8 & 0.3 & 24.5 & 0.3 & $24 \cdot 2$ & 0.3 & $24 \cdot 7$ & 0.3 & 0.93 \\
\hline Maltose & 24.9 & 0.3 & $24 \cdot 8$ & 0.3 & $24 \cdot 7$ & 0.3 & 24.5 & 0.3 & $24 \cdot 6$ & 0.3 & 0.57 \\
\hline Sugar-sweetened beverages & $25 \cdot 1$ & 0.3 & $25 \cdot 1$ & $0 \cdot 3$ & $24 \cdot 5$ & $0 \cdot 3$ & $24 \cdot 6$ & $0 \cdot 3$ & 24.4 & 0.3 & 0.005 \\
\hline Sugar-sweetened solid foods & 24.9 & 0.3 & 24.9 & 0.3 & $24 \cdot 8$ & 0.3 & $24 \cdot 1$ & 0.3 & $25 \cdot 1$ & 0.3 & 0.50 \\
\hline $100 \%$ fruit juice & 24.5 & 0.3 & $25 \cdot 0$ & 0.3 & $24 \cdot 7$ & 0.3 & $25 \cdot 1$ & 0.3 & $24 \cdot 1$ & 0.3 & 0.35 \\
\hline
\end{tabular}

* Mean value was significantly different from that of the first quintile $(P<0.05)$.

† Values were adjusted for age (years), sex, educational attainment ( $<5$ th grade, 5 th -8 th grade, 9th-12th grade, college, or graduate school), poverty (yes or no), acculturation score, smoking (never, former, or current), alcohol use (not current, current moderate, current heavy), physical activity score, BMI (kg/m²; $<25,25-$ 29.9 , or $\geq 30$ ) and the presence of hypertension. Means were compared by using the general linear model procedure in SAS, with Tukey's adjustment for multiple comparisons.

$\ddagger$ Continuous value of sugar consumption was used for the trend test

$\S$ Non-added fructose and added fructose were included in the model simultaneously.

score was $0 \cdot 4$ points lower for each $50 \mathrm{~g}$ of added sugar intake ( $\beta-0.43$; sE $0 \cdot 16 ; P=0.005$ ), which also equalled the difference in MMSE score per 10-year increase in age $(\beta-0.43$; SE $0 \cdot 17 ; P=0 \cdot 011)$. These associations were attenuated after further adjustment for intakes of dietary fibre and fat (saturated, polyunsaturated and trans), vitamin supplement use and serum concentrations of vitamin $\mathrm{C}$, total carotenoids and total homocysteine, but still remained significant for total sugar ( $P$ for trend=0.024) and marginally significant for added sugar ( $P$ for trend $=0 \cdot 057$ ).

Similar inverse trends were observed for sucrose, glucose and fructose, after adjusting for covariates (Table 3). Further analysis showed that added fructose, but not natural fructose, was significantly associated with lower MMSE score. The association between sucrose and MMSE score was attenuated after further adjustment for the sum of glucose, fructose, galactose, lactose and maltose ( $P$ for trend=0.059). The associations between glucose ( $P$ for trend $=0.083$ ), as well as fructose $(P$ for trend $=0.093$ ) and added fructose ( $P$ for trend $=0.088$, and MMSE score were also attenuated after further adjustment for the sum of sucrose, galactose, lactose and maltose.

No significant associations between intakes of galactose, lactose or maltose and MMSE score were observed (Table 3). SSB, but not sugar-sweetened solid foods or 100\% fruit juice, were inversely associated with MMSE score. Higher intakes of total and added sugars were also associated with a higher likelihood of having cognitive impairment (MMSE score <24) (Fig. 1); the multiple-adjusted OR for cognitive impairment were $2 \cdot 23$ (95\% CI 1.24, 3.99) and 2.28 (95\% CI $1 \cdot 26,4 \cdot 14)$ for the highest $v$. lowest quintiles of total and added sugar consumption, respectively. A higher intake of fructose was also marginally associated with lower cognitive impairment (OR 1.57; 95\% CI 0.91, 2.73) when comparing extreme quintiles ( $P$ for trend=0.072). The relative OR for cognitive impairment defined by a more conservative cut-off point (MMSE score <21) were 1.71 (95\% CI $0.81,3.61$ ) for total sugars ( $P$ for trend=0.029), 2.42 (95\% CI $1 \cdot 08,5 \cdot 39)$ for added sugars ( $P$ for trend=0.029), and 1.43 (95\% CI 0.70 , 2.93) for fructose ( $P$ for trend $=0.078$ ) when comparing extreme quintiles, respectively.

Total sugar intakes were inversely associated with memory function and a similar trend with marginal significance was observed for added sugar intakes (Table 4). Inverse associations were also observed for total sugar intake with letter fluency and word list percentage recognition, delayed recognition, short- and long-term recall scores; and for added sugar intake with letter fluency and word list long-term recall score (all $P<0.05$ ) (data not shown).

\section{Discussion}

In this sample of Puerto Rican adults without diabetes, aged 45-75 years, approximately $21 \%$ of energy intake was obtained from total sugars and 12\% from added sugars. Total sugars, added sugars, sucrose, glucose and added fructose were each significantly inversely associated with cognitive function. SSB, but not sugar-sweetened solid foods, were inversely associated with cognitive function. Higher intakes of total sugars were also significantly associated with lower memory function, but not with measurable differences in executive function or attention.

The percentage contribution of added sugar to total energy intake in the present study was similar to that of the general US population with a similar age range, as shown in the 1994-6 Continuing Survey of Food Intakes by Individuals $(\mathrm{CSFII})^{(39)}$ and the National Health and Nutrition Examination Survey (NHANES 1999-2002) ${ }^{(40)}$. However, in the 1994-6 


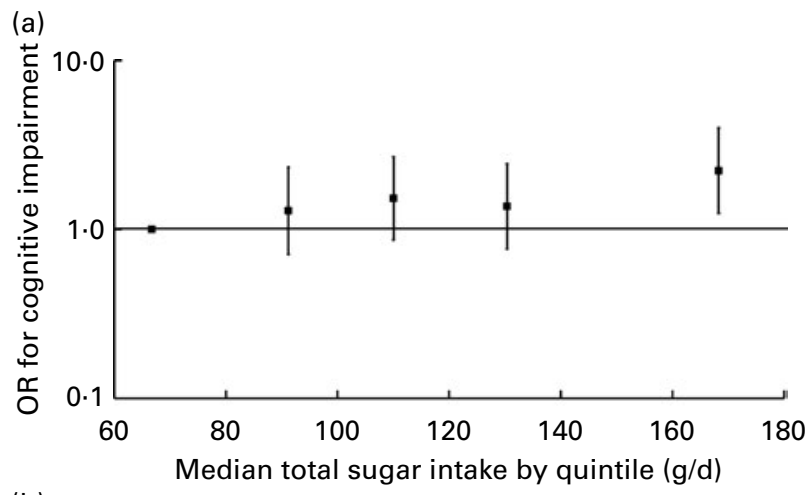

(b)

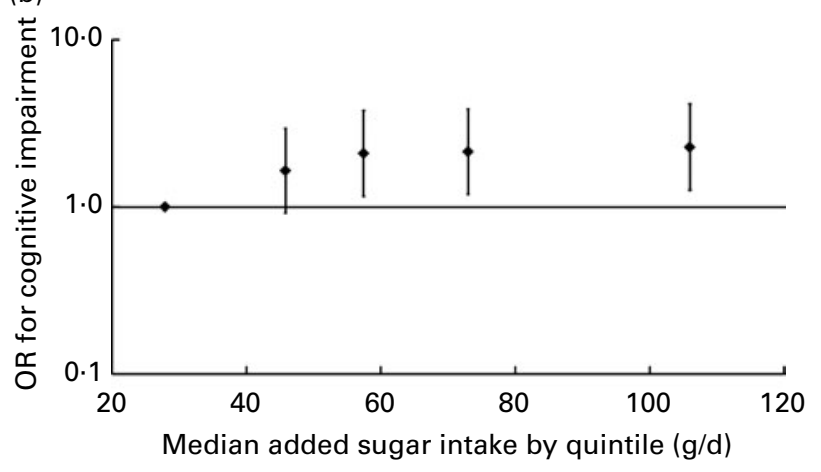

(c)

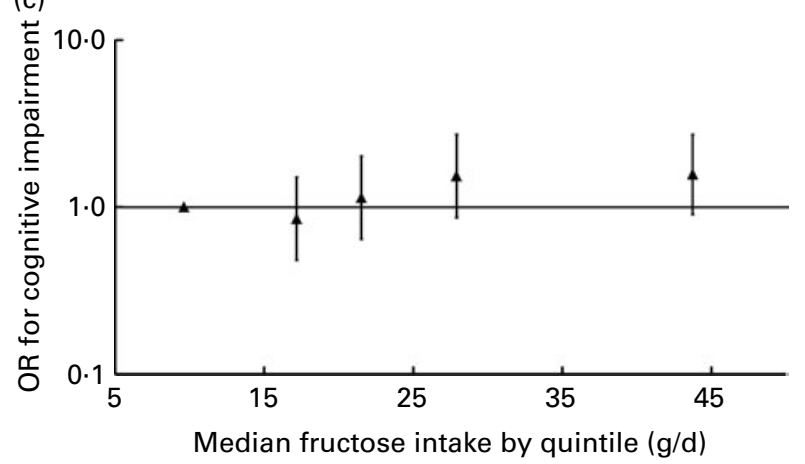

Fig. 1. Multivariate-adjusted $\mathrm{OR}$ and $95 \% \mathrm{Cl}$ for cognitive impairment according to quintile of energy-adjusted total sugar ( $P$ for trend=0.015) (a), added sugar intakes $(P$ for trend $=0.040)(b)$ and fructose intake (natural plus added) $(P$ for trend $=0.072$ ) (c) among participants without diabetes from the Boston Puerto Rican Health Study, 2004-9. OR and $95 \% \mathrm{Cl}$ were plotted against the median of sugar intake of each quintile. The median (range) of total sugar intake $(\mathrm{g} / \mathrm{d})$ across quintiles of total sugar intake were: 66.7 (<82.0), 91.2 (82.0-100.8), 110.0 (100.9-118.3), 130.4 (118.4-145.5) and 168.3 (>145.5), respectively. The median (range) of added sugar intake $(\mathrm{g} / \mathrm{d})$ across quintiles of added sugar intake were: $27.9(<37.9), 45.8(38.0-$ $51.0), 57.4(51.1-64.6), 73.0(64.7-85.1)$ and 106.0 (>85.1), respectively. The median (range) of fructose intake $(\mathrm{g} / \mathrm{d})$ across quintiles of fructose intake were: 9.6 (<14.2), 17.2 (14.2-19.3), 21.5 (19.4-24.9), 27.9 (25.0-33.8) and 43.7 (>33.8), respectively. Cognitive impairment was defined as a MiniMental State Examination score $<24$. OR was calculated with logistic regression, after adjustment for age (years), sex, educational attainment ( $<5$ th grade, 5 th-8th grade, 9th-12th grade, college, or graduate school), poverty (yes or no), acculturation score, smoking (never, former, or current), alcohol use (never, former, or current), physical activity score, BMI $\left(\mathrm{kg} / \mathrm{m}^{2}\right.$; $<25,25-29 \cdot 9$, or $\geq 30$ ), and the presence of hypertension.

CSFII, soft drinks provided $33 \%$ of total added sugars, and fruit drinks provided $10 \%{ }^{(39)}$; and in the NHANES 2001-2, soft drinks provided $37 \%$ and fruit drinks $12 \%{ }^{(41)}$. In contrast, the percentage contributions of added sugars from fruit drinks were higher than from soft drinks in the present study population.

The mean MMSE score (23.7) was generally low when compared with other studies. For example, in the Northern Manhattan Study of 3298 stroke-free subjects aged 40 years and older, the mean MMSE score for Hispanic subjects was $25 \cdot 3$, which was significantly lower than that for white subjects $(27 \cdot 7)^{(42)}$. The low mean MMSE score and high prevalence of cognitive impairment (MMSE score <24) in the present study may be due to differences in language use, low educational attainment ${ }^{(36)}$ and high prevalence of risk factors and diseases that may affect cognitive function.

Previous studies have shown that high sugar consumption is a risk factor for obesity ${ }^{(3,4)}$, the metabolic syndrome ${ }^{(5,8)}$ and type 2 diabetes ${ }^{(4,8-10,43,44)}$. Obesity, the metabolic syndrome and type 2 diabetes are risk factors for cognitive impairment $^{(12-15,45)}$. Cognitive impairment in type 2 diabetes has been more evident for verbal memory than for attention, concentration or executive function ${ }^{(45)}$. We observed that higher sugar consumption was more significantly associated with lower memory function, than with other cognitive measures. Findings from animal studies have also suggested that diets with high sugar may reduce memory function ${ }^{(17,46)}$. For example, Cao et al. $^{(46)}$ found that long-term consumption of sucrose-sweetened water induced insulin resistance and exacerbated memory deficits in a transgenic mouse model of Alzheimer's disease. Stranahan et $a l^{(17)}$ showed that rats fed high-fructose diets developed insulin resistance, resulting in impaired hippocampal synaptic plasticity and hippocampusdependent memory. More recently, Rasgon et al. ${ }^{(47)}$ reported that insulin resistance was inversely associated with right and total hippocampal volume among non-diabetic, cognitively intact middle-aged women at risk for Alzheimer's disease $^{(47)}$.

Nevertheless, an inverse association between BMI and total sugar intake observed in the present study deserves attention, and could be explained by several possible mechanisms. In a 10 -week intervention in overweight subjects, Raben et al. ${ }^{\text {(48) }}$ showed that those in the sucrose-supplemented group had an increased fat mass of $1.3 \mathrm{~kg}$ (total weight increased by $1.6 \mathrm{~kg}$ ), compared with those receiving artificial sweetener, where fat mass decreased by $0.3 \mathrm{~kg}$ (total weight decreased by $1.0 \mathrm{~kg})^{(48)}$. These results suggest that sucrose may contribute more to change in body fat than in total weight. Because BMI has its own limitation in assessing fatness among elder individuals $^{(49)}$, further studies are needed to verify whether sugar intake is more correlated to body fat than to BMI among older individuals. We also cannot exclude the possibility that those with higher BMI may under-report sugar intake and sweetened food and beverages than their counterparts ${ }^{(50)}$. Those with the highest BMI were younger in the present study, and it is possible that they may have been more concerned about their body weight than their older counterparts, and may have tried to reduce their sugar intake. Prospective studies are needed to verify the role of sugar intake in the development of obesity in this population.

Importantly, however, the observed associations between sugar intake and MMSE were independent of age and BMI. 
Table 4. Major cognitive function factors* by quintile of intake of energy-adjusted total sugars and added sugars in participants without diabetes from the Boston Puerto Rican health study, 2004-9†

(Mean values with their standard errors)

\begin{tabular}{|c|c|c|c|c|c|c|c|c|c|c|c|}
\hline & \multicolumn{2}{|c|}{ 1st quintile } & \multicolumn{2}{|c|}{ 2nd quintile } & \multicolumn{2}{|c|}{ 3rd quintile } & \multicolumn{2}{|c|}{ 4th quintile } & \multicolumn{2}{|c|}{ 5th quintile } & \multirow{2}{*}{$\begin{array}{l}P \text { for } \\
\text { trend }\end{array}$} \\
\hline & Mean & SE & Mean & SE & Mean & SE & Mean & SE & Mean & SE & \\
\hline \multicolumn{12}{|l|}{ Total sugars } \\
\hline Memory & 0.18 & 0.11 & 0.08 & 0.11 & 0.21 & 0.11 & 0.02 & 0.11 & 0.05 & 0.11 & 0.010 \\
\hline Attention & 0.35 & 0.11 & 0.42 & 0.11 & 0.25 & 0.10 & 0.28 & 0.11 & 0.11 & $0 \cdot 11$ & 0.062 \\
\hline Executive function & -0.10 & 0.09 & 0.13 & 0.10 & -0.02 & 0.09 & 0.15 & $0 \cdot 10$ & 0.06 & $0 \cdot 10$ & 0.17 \\
\hline \multicolumn{12}{|l|}{ Added sugars } \\
\hline Memory & $0 \cdot 21$ & $0 \cdot 10$ & $0 \cdot 15$ & $0 \cdot 11$ & 0.03 & $0 \cdot 11$ & 0.09 & $0 \cdot 11$ & 0.03 & $0 \cdot 11$ & 0.072 \\
\hline Attention & 0.42 & 0.10 & 0.30 & 0.10 & 0.15 & 0.11 & 0.26 & 0.11 & 0.19 & $0 \cdot 11$ & 0.10 \\
\hline Executive function & 0.05 & 0.10 & -0.08 & 0.09 & 0.12 & 0.10 & 0.08 & $0 \cdot 10$ & 0.03 & $0 \cdot 10$ & 0.57 \\
\hline
\end{tabular}

* Three major cognitive function factors were derived from seven cognitive tests by factor analysis (see Supplemental Table 1; available at http://www.journals. cambridge.org/bjn).

$\dagger$ Values were adjusted for age (years), sex, educational attainment ( $<5$ th grade, 5th-8th grade, 9th-12th grade, college, or graduate school), poverty (yes or no), acculturation score, smoking (never, former, or current), alcohol use (not current, current moderate, current heavy), physical activity score, BMl (kg/m²; $<25$, $25-29 \cdot 9$, or $\geq 30$ ) and the presence of hypertension. Means were compared by using the general linear model procedure in SAS, with Tukey's adjustment for multiple comparisons.

Sugar intake and cognitive function may also be connected through other mechanisms ${ }^{(21)}$. Sucrose and HFCS have been shown to increase the production of uric acid in human subjects ${ }^{(51)}$. An association between elevated uric acid and cognitive decline was recently reported in older individuals ${ }^{(52,53)}$. In addition, a dietary pattern with high SSB was related to elevated inflammatory status ${ }^{(54)}$. A 10 -week intervention study demonstrated that sugar-sweetened drinks and food tend to increase inflammatory activity ${ }^{(5)}$. Inflammation, a major risk factor for diabetes and CVD, is also correlated with cognitive decline $^{(56,57)}$ and dementia ${ }^{(58)}$ even after adjustment for cardiovascular metabolic disorders. Fructose, one of the major ingredients of SSB, has been linked to increased de novo lipogenesis, glycogenesis, oxidative stress and uric acid production, and to reduced NO production ${ }^{(21)}$. As reviewed by Stephan et $a l^{(21)}$, these metabolic changes have been associated with the metabolic syndrome or its components which, in turn, may lead to cognitive decline and Alzheimer's disease ${ }^{(11-14,59,60)}$

Interestingly, added fructose, but not natural fructose, was related to low MMSE scores. Natural fructose is from fruit and vegetables, which also contribute protective nutrients for cognitive function, including $\mathrm{B}$ vitamins and antioxidants ${ }^{(61,62)}$, whereas added fructose is mainly added to SSB. Importantly, $100 \%$ fruit juice, unlike SSB, was not associated with lower MMSE score or memory function. Other nutrients in $100 \%$ fruit juice have been positively linked with cognitive function $^{(61,62)}$ and, therefore, may counteract the potential effects of naturally occurring sugars in $100 \%$ fruit juice.

It is important to note that SSB, but not sugar-sweetened solid foods, were associated with lower cognitive function. One potential interpretation could be the differential contributions of sucrose, glucose and fructose in beverages $v$. solid foods. SSB were strongly associated with intakes of glucose and added fructose, whereas sugar-sweetened solid foods were only associated with the total intake of sucrose.

Intake of total sugars was independently associated with lower cognitive function, after adjusting for supplemental use of vitamins, dietary fibre and fat, and plasma concentrations of several nutrients as was the association of intake of added sugars with cognitive function, although marginally significant ( $P$ for trend $=0 \cdot 065$ ). These findings suggest that the inverse associations between sugar consumption and cognitive function were not fully explained by other aspects of a poorer dietary pattern that may be associated with higher sugar consumption.

Alternatively, our findings could also be explained by potentially altered dietary selections because of chemosensory changes with ageing and cognitive decline ${ }^{(63)}$. Older participants have been reported to have a preference for higher concentrations of sugar relative to their younger counterparts ${ }^{(63)}$. A high proportion of participants had evidence of cognitive impairment (MMSE score <24) in the present study. Some of these low scores are probably related to low education levels ${ }^{(64)}$. Although we excluded those with MMSE score $\leq 10$, it is possible that some with scores between 10 and 24 could, if clinically diagnosed, be found to have mild cognitive impairment or early-stage Alzheimer's disease. A few studies have suggested that patients with Alzheimer's disease may have a greater preference for sweet foods than non-impaired controls $^{(65)}$. Due to the cross-sectional design of available studies, prospective studies are needed to clarify causal associations between sweet foods and cognitive decline.

The present study has several strengths. We conducted analyses among individuals without diabetes, which reduces the potential confounding effects of diabetes and its complications, and other important confounding factors were controlled. We used a series of cognitive function tests that allowed the assessment of different domains of cognitive function. Limitations include the cross-sectional design, which precludes conclusions regarding the direction of causality. We were also not able to separate the specific associations of fructose with cognitive function from glucose because they were highly correlated with each other. Further, although we controlled for several possible confounders in our analyses, we cannot exclude the possibility of residual confounding.

To our knowledge, this is the first population-based study to simultaneously investigate the associations between habitual 
intakes of sugars, SSB and sugar-sweetened solid foods with cognitive function among middle-aged and older adults. Total sugars, added sugars, sucrose and added fructose were each inversely associated with cognitive performance. Consistently, a higher intake of SSB was significantly associated with lower cognitive performance. Prospective studies are needed to confirm whether long-term consumption of SSB and/or sugar-sweetened foods is a risk factor for cognitive impairment.

\section{Acknowledgements}

The present study was supported by the National Institute on Aging of the National Institutes of Health (NIH) (no. P01AG023394 and R01AG02708), the National Heart Lung and Blood Institute of NIH (no. P50HL105185) and the US Department of Agriculture, Agricultural Research Service contract (no. 58-1950-7-707).

X. Y. contributed to data analysis, interpretation of data and writing the manuscript. X. G., T. S. and K. L. T contributed to the interpretation of data and critical revision of the manuscript. K. L. T obtained funding, conceived of the study and supervised the data collection. All authors read and approved the final manuscript.

None of the authors had any conflicts of interest.

\section{References}

1. Nielsen SJ \& Popkin BM (2004) Changes in beverage intake between 1977 and 2001. Am J Prev Med 27, 205-210.

2. Wang Y \& Beydoun MA (2007) The obesity epidemic in the United States - gender, age, socioeconomic, racial/ethnic, and geographic characteristics: a systematic review and meta-regression analysis. Epidemiol Rev 29, 6-28.

3. Forshee RA, Storey ML, Allison DB, et al. (2007) A critical examination of the evidence relating high fructose corn syrup and weight gain. Crit Rev Food Sci Nutr 47, 561-582.

4. Bleich SN, Wang YC, Wang Y, et al. (2009) Increasing consumption of sugar-sweetened beverages among US adults: 1988-1994 to 1999-2004. Am J Clin Nutr 89, 372-381.

5. Dhingra R, Sullivan L, Jacques PF, et al. (2007) Soft drink consumption and risk of developing cardiometabolic risk factors and the metabolic syndrome in middle-aged adults in the community. Circulation 116, 480-488.

6. Chen L, Caballero B, Mitchell DC, et al. (2010) Reducing consumption of sugar-sweetened beverages is associated with reduced blood pressure: a prospective study among United States adults. Circulation 121, 2398-2406.

7. Jalal DI, Smits G, Johnson RJ, et al. (2010) Increased fructose associates with elevated blood pressure. J Am Soc Nephrol 21, 1543-1549.

8. Malik VS, Popkin BM, Bray GA, et al. (2010) Sugarsweetened beverages and risk of metabolic syndrome and type 2 diabetes: a meta-analysis. Diabetes Care 33, 2477-2483.

9. Schulze MB, Manson JE, Ludwig DS, et al. (2004) Sugarsweetened beverages, weight gain, and incidence of type 2 diabetes in young and middle-aged women. JAMA 292, 927-934.

10. Odegaard AO, Koh WP, Arakawa K, et al. (2010) Soft drink and juice consumption and risk of physician-diagnosed incident type 2 diabetes: the Singapore Chinese Health Study. Am J Epidemiol 171, 701-708.
11. Dik MG, Jonker C, Comijs HC, et al. (2007) Contribution of metabolic syndrome components to cognition in older individuals. Diabetes Care 30, 2655-2660.

12. Yaffe K, Kanaya A, Lindquist K, et al. (2004) The metabolic syndrome, inflammation, and risk of cognitive decline. JAMA 292, 2237-2242.

13. Yaffe K, Haan M, Blackwell T, et al. (2007) Metabolic syndrome and cognitive decline in elderly Latinos: findings from the Sacramento Area Latino Study of Aging study. J Am Geriatr Soc 55, 758-762.

14. Ho RC, Niti M, Yap KB, et al. (2008) Metabolic syndrome and cognitive decline in Chinese older adults: results from the singapore Longitudinal Ageing Studies. Am J Geriatr Psychiatry 16, 519-522.

15. van den Berg E, Kloppenborg RP, Kessels RPC, et al. (2009) Type 2 diabetes mellitus, hypertension, dyslipidemia and obesity: a systematic comparison of their impact on cognition. Biochim Biophys Acta 1792, 470-481.

16. Jones JM (2009) Dietary sweeteners containing fructose: overview of a workshop on the state of the science. $J$ Nutr 139, 1210S-1213S.

17. Stranahan AM, Norman ED, Lee K, et al. (2008) Diet-induced insulin resistance impairs hippocampal synaptic plasticity and cognition in middle-aged rats. Hippocampus $\mathbf{1 8}$, 1085-1088.

18. Mielke JG, Taghibiglou C, Liu L, et al. (2005) A biochemical and functional characterization of diet-induced brain insulin resistance. J Neurochem 93, 1568-1578.

19. White JS (2009) Misconceptions about high-fructose corn syrup: is it uniquely responsible for obesity, reactive dicarbonyl compounds, and advanced glycation endproducts? J Nutr 139, 1219S-1227S.

20. Gilsenan MB, de Bruin EA \& Dye L (2009) The influence of carbohydrate on cognitive performance: a critical evaluation from the perspective of glycaemic load. Br J Nutr 101, 941-949.

21. Stephan BC, Wells JC, Brayne C, et al. (2010) Increased fructose intake as a risk factor for dementia. $J$ Gerontol $A$ Biol Sci Med Sci 65, 809-814.

22. Tucker K, Mattei J, Noel S, et al. (2010) The Boston Puerto Rican Health Study, a longitudinal cohort study on health disparities in Puerto Rican adults: challenges and opportunities. BMC Public Health 10, 107.

23. Noel SE, Newby PK, Ordovas JM, et al. (2009) A traditional rice and beans pattern is associated with metabolic syndrome in Puerto Rican older adults. J Nutr 139, 1360-1367.

24. Bermudez OI, Ribaya-Mercado JD, Talegawkar SA, et al. (2005) Hispanic and non-Hispanic white elders from Massachusetts have different patterns of carotenoid intake and plasma concentrations. J Nutr 135, 1496-1502.

25. Kwan LL, Bermudez OI \& Tucker KL (2002) Low vitamin B-12 intake and status are more prevalent in Hispanic older adults of Caribbean origin than in neighborhoodmatched non-Hispanic whites. J Nutr 132, 2059-2064.

26. Institute of Medicine of the National Academies (2002) Dietary Reference Intakes: Energy, Carbohydrate, Fiber, Fat, Fatty Acids, Cholesterol, Protein, and Amino Acids. Washington, DC: The National Academies Press.

27. Gao X, Scott T, Falcon LM, et al. (2009) Food insecurity and cognitive function in Puerto Rican adults. Am J Clin Nutr 89, 1197-1203.

28. Folstein MF, Folstein SE \& McHugh PR (1975) "Mini-mental state". A practical method for grading the cognitive state of patients for the clinician. J Psychiatr Res 12, 189-198.

29. Artiola Fortuny L, Hermosillo Romo D, Heaton RK, et al. (2000) Manual de Normas y Procedimientos para la Batería 
Neuropsicológica en Español (Policies and Procedures Manual for Neuropsychological Battery in Spanish). Brookfield, VT: Swets \& Zeitlinger

30. Wolf-Klein GP, Silverstone FA, Levy AP, et al. (1989) Screening for Alzheimer's disease by clock drawing. J Am Geriatr Soc 37, 730-734.

31. Beery K (1989) The Developmental Test of Visual-Motor Integration Manual, revised ed. Cleveland, OH: Modern Curriculum Press.

32. Kaiser HF (1960) The application of electronic computers to factor analysis. Educ Psychol Meas 20, 141-151.

33. Paffenbarger RS, Hyde RT, Wing AL, et al. (1993) The association of changes in physical-activity level and other lifestyle characteristics with mortality among men. $N$ Engl J Med $\mathbf{3 2 8}$, 538-545.

34. Falcon LM \& Tucker KL (2000) Prevalence and correlates of depressive symptoms among Hispanic elders in Massachusetts. J Gerontol B Psychol Sci Soc Sci 55, S108-S116.

35. Willett WC, Howe GR \& Kushi LH (1997) Adjustment for total energy intake in epidemiologic studies. Am J Clin Nutr $\mathbf{6 5}$, S1220S-S1231S.

36. Escobar JI, Burnam A, Karno M, et al. (1986) Use of the MiniMental State Examination (MMSE) in a community population of mixed ethnicity. Cultural and linguistic artifacts. $J$ Nerv Ment Dis 174, 607-614.

37. Ayala GX, Baquero B \& Klinger S (2008) A systematic review of the relationship between acculturation and diet among Latinos in the United States: implications for future research. $J$ Am Diet Assoc 108, 1330-1344.

38. Robison J, Gruman C, Gaztambide S, et al. (2002) Screening for depression in middle-aged and older Puerto Rican primary care patients. J Gerontol A Biol Sci Med Sci 57, M308-M314.

39. Guthrie JF \& Morton JF (2000) Food sources of added sweeteners in the diets of Americans. J Am Diet Assoc 100, 43-51.

40. Cook A \& Friday J (2005) CNRG Table Set 3.0: Pyramid Servings Intakes in the United States, 1999-2002, 1 Day. USDA, Agricultural Research Service. http://www.ars.usda.gov/ sp2UserFiles/place/12355000/foodlink/ts_3-0.pdf/

41. Bachman JL, Reedy J, Subar AF, et al. (2008) Sources of food group intakes among the US population, 2001-2002. J Am Diet Assoc 108, 804-814.

42. Wright CB, Lee H-S, Paik MC, et al. (2004) Total homocysteine and cognition in a tri-ethnic cohort: The Northern Manhattan Study. Neurology 63, 254-260.

43. Laville M \& Nazare J-A (2009) Diabetes, insulin resistance and sugars. Obes Rev 10, 24-33.

44. Johnson RJ, Segal MS, Sautin Y, et al. (2007) Potential role of sugar (fructose) in the epidemic of hypertension, obesity and the metabolic syndrome, diabetes, kidney disease, and cardiovascular disease. Am J Clin Nutr 86, 899-906.

45. Greenwood CE (2003) Dietary carbohydrate, glucose regulation, and cognitive performance in elderly persons. Nutr Rev 61, S68-S74.

46. Cao D, Lu H, Lewis TL, et al. (2007) Intake of sucrosesweetened water induces insulin resistance and exacerbates memory deficits and amyloidosis in a transgenic mouse model of Alzheimer disease. $J$ Biol Chem 282, 36275-36282.

47. Rasgon NL, Kenna HA, Wroolie TE, et al. (2009) Insulin resistance and hippocampal volume in women at risk for
Alzheimer's disease. Neurobiol Aging (epublication 25 December 2009).

48. Raben A, Vasilaras TH, Moller AC, et al. (2002) Sucrose compared with artificial sweeteners: different effects on ad libitum food intake and body weight after $10 \mathrm{wk}$ of supplementation in overweight subjects. Am J Clin Nutr 76, 721-729.

49. Kyle UG, Genton L, Hans D, et al. (2001) Age-related differences in fat-free mass, skeletal muscle, body cell mass and fat mass between 18 and 94 years. Eur J Clin Nutr $\mathbf{5 5}$, 663-672.

50. Mendez MA, Wynter S, Wilks R, et al. (2004) Under- and overreporting of energy is related to obesity, lifestyle factors and food group intakes in Jamaican adults. Public Health Nutr 7, 9-19.

51. Akhavan T \& Anderson GH (2007) Effects of glucose-tofructose ratios in solutions on subjective satiety, food intake, and satiety hormones in young men. Am J Clin Nutr 86, 1354-1363.

52. Schretlen DJ, Inscore AB, Jinnah HA, et al. (2007) Serum uric acid and cognitive function in community-dwelling older adults. Neuropsychology 21, 136-140.

53. Ruggiero C, Cherubini A, Lauretani F, et al. (2009) Uric acid and dementia in community-dwelling older persons. Dement Geriatr Cogn Disord 27, 382-389.

54. Schulze MB, Hoffmann K, Manson JE, et al. (2005) Dietary pattern, inflammation, and incidence of type 2 diabetes in women. Am J Clin Nutr 82, 675-684.

55. Sorensen LB, Raben A, Stender S, et al. (2005) Effect of sucrose on inflammatory markers in overweight humans. Am J Clin Nutr 82, 421-427.

56. Weaver JD, Huang MH, Albert M, et al. (2002) Interleukin-6 and risk of cognitive decline: MacArthur studies of successful aging. Neurology 59, 371-378.

57. Yaffe K, Lindquist K, Penninx BW, et al. (2003) Inflammatory markers and cognition in well-functioning African-American and white elders. Neurology 61, 76-80.

58. Engelhart MJ, Geerlings MI, Meijer J, et al. (2004) Inflammatory proteins in plasma and the risk of dementia: the Rotterdam Study. Arch Neurol 61, 668-672.

59. Vanhanen M, Koivisto K, Moilanen L, et al. (2006) Association of metabolic syndrome with Alzheimer disease: a population-based study. Neurology 67, 843-847.

60. Razay G, Vreugdenhil A \& Wilcock G (2007) The metabolic syndrome and Alzheimer disease. Arch Neurol 64, 93-96.

61. Tucker KL, Qiao N, Scott T, et al. (2005) High homocysteine and low $\mathrm{B}$ vitamins predict cognitive decline in aging men: the Veterans Affairs Normative Aging Study. Am J Clin Nutr 82, 627-635.

62. Akbaraly NT, Faure H, Gourlet V, et al. (2007) Plasma carotenoid levels and cognitive performance in an elderly population: results of the EVA Study. J Gerontol A Biol Sci Med Sci 62, 308-316.

63. Murphy C (1993) Nutrition and chemosensory perception in the elderly. Crit Rev Food Sci Nutr 33, 3-15.

64. Crum RM, Anthony JC, Bassett SS, et al. (1993) Populationbased norms for the Mini-Mental State Examination by age and educational level. JAMA 269, 2386-2391.

65. Mungas D, Cooper JK, Weiler PG, et al. (1990) Dietary preference for sweet foods in patients with dementia. $J \mathrm{Am}$ Geriatr Soc 38, 999-1007. 